\title{
The initial effect of noise on a simple vibrotactile learning task'
}

\author{
D. D. DIESPECKER AND W. G. DAVENPORT \\ UNIVERSITY OF NEWCASTLE, N.S.W., AUSTRALIA
}

In two experiments the effect of loud noise on a simple vibrotactile learning task was studied. After learning the task to criterion, 10 male and 10 female $S$ s received two pairs of test trials, one without noise, and one in continuous noise (a 1,000 cps pure tone at $90 \mathrm{~dB}$ SPL). An additional 10 male and 10 female Ss leamed the same task and also received the same two pairs of test trials, but instead of receiving continuous noise for the second condition, they received an intermittent noise (random numbers presented at $2 \mathrm{sec}$ intervals at $95 \mathrm{~dB}$ SPL). In the first experiment no ise had a significant effect on the performance of the 20 subject group and also on the females in the group. Noise did not significantly affect the performance of males. In the second experiment noise had no significant effect on either males or females.

The effect of loud noises on learning and perceptual tasks is controversial.

Brown et al (1965) have cited some of the relevant literature in this area and they have also drawn attention to the lack of research for studying specifically the effects of extraneous variables on cutaneous information processing. One of these variables, intense noise, was considered to be particularly important. Brown and his associates transmitted electropulse signals (three levels of message complexity) to Ss under three conditions of noise: intermittent noise, continuous noise, and no noise. They found that intense auditory noise had little effect upon the reception and processing of cutaneously presented information.

Although electropulse stimuli are now receiving a great deal of research attention, most of the data pertaining to cutaneous communication have been obtained by using mechanical rather than electrical forms of energy (Geldard, 1960, 1961, 1962; Gilmer, 1966). The most intensively studied form of energy has been pressure, i.e., mechanical vibratory or vibrotactile stimuli.

The present studies were made to investigate the effect of loud noises on a simple vibrotactile learning task. The initial effect of noise on such a task was considered to be especially important since the Brown et al conclusion was based on results from 50-trial experimental programs.

The first experiment reported here investigated the effect of high intensity continuous noise (90 dB SPL pure tone at $1000 \mathrm{cps}$ ) on a vibrotactile learning task. The second experiment was made to in- vestigate the effect of intermittent noise (a recording of random numbers at $95 \mathrm{~dB}$ SPL) on the same vibrotactile learning task.

\section{EXPERIMENT 1}

Pure Tone (90 dB re 0.0002 dyne $/ \mathrm{cm}^{2}$ )

\section{Apparatus}

Signals were transmitted to a modified Sherrick transducer (Sherrick, 1965) attached to the left forearm of $S$. The transducer was cylindrical rather than cubical in form. The coil form was a 1 in. long Perspex tube with a wall thickness of $1 / 8 \mathrm{in.}$ and a 1 in. diameter. A Rola Company ferrite magnet, $3 / 4$ in. $x 3 / 4$ in. $x 1 / 2$ in., was packed in foam plastic and sealed inside the coil form. The contactor was circular Perspex 1/16 in. thick having an area of 1.9 in. sq. The coil form was wound with 800 turns of $0.010 \mathrm{in}$. diameter enamelled magnet wire.

A small control unit was used to energize the vibrator (Hodge \& Diespecker, 1967). It transmitted signals having three electrical intensities: $10 \mathrm{~V}, 15 \mathrm{~V}$, and $20 \mathrm{~V}$. This unit was also connected to a decade interval timer. Three durations of signal were selected as required: $0.1 \mathrm{sec}, 0.3 \mathrm{sec}$, and $0.5 \mathrm{sec}$. The apparatus used to produce vibrotactile signals thus allowed a nine-element coding system to be utilized in the investigation.

Auditory signals were obtained from an Advance 81A LF signal generator and a Marconi TF 2161 MF monitored attenuator.

Vibrotactile signals were transmitted by wire to the transducer while $S$ sat in a chair with arm rests. An exhaust fan was used during training and test trials to mask any noise from the operating equipment. At no time could the vibrotactile signals be seen or heard.

The experiment was conducted in an otherwise quiet laboratory which was free of building vibration.

\section{Subjects}

The Ss were 10 male and 10 female undergraduate students in the University of Newcastle, New South Wales.

\section{Procedure}

The threshold for hearing was determined for each $S$ (method of limits).

Ss were tested singly. S was seated comfortably in the experimental chair and the design of the experiment was described by $E$. A nine-element 
Table 1: Vibrotactile Code.

\begin{tabular}{lccc} 
& Short & Medium & Long \\
\hline Hord & 3 & 6 & 9 \\
Medium & 2 & 5 & 8 \\
Soft & 1 & 4 & 7
\end{tabular}

vibrotactile code was tabulated on a blackboard in the laboratory and was visible to $s$ throughout the experiment. The code is given in Table 1.

After two demonstrations of each signal in serial order (without naming the numerical equivalents of the signals) $E$ presented $S$ with a series of training trials. Each trial consisted of nine "numbers" in random order (the numbers 1-9). After the transmission of a signal $S$ was required to respond by naming a number. $E$ then announced the correct signal and repeated it whether it had been correctly identified or not. The same procedure was employed in test trials in both experiments.

In the first part of the experiment $S$ was required to reach a criterion of one correct training trial.

Trials were continuous except in one instance when a second session was required to complete training. There was no fixed interstimulus interval, but the signals were approximately 5 sec apart.

When S had achieved $100 \%$ correct responses two pairs of test trials were given. Each pair consisted of 18 numbers, but the digits were randomized between the two trials. Trial $A$, for example, consisted of the numbers $2,7,6,6,7,1,8,1,3$ and Trial $B$ comprised the numbers $2,4,8,3,9,4,9,5,5$.

$S$ was given a pair of test trials in two conditions of noise: no noise and loud noise, in this order. Each $S$ received a pure tone of $90 \mathrm{~dB}$ intensity (re 0.0002 dyne $/ \mathrm{cm}^{2}$ ) during noise trials. The signal was continuous.

\section{Resulis}

The frequencies of correct responses for male and female Ss under both conditions of noise are given in Table 2. The data were analyzed by $t$ tests. Female performance was significantly worse in noise than in no-noise $(t=2.82, \mathrm{df}=9, \mathrm{p}<0.05)$. However, male performance was not significantly affected by the noise $(t=0.18, d f=9, p>0.05)$. Combining the male and female scores showed that noise again had a significant adverse effect on performance $(t=2.25$, $d f=19, p<0.05$ ).

\section{EXPERIMENT 2}

Random Numbers

\section{Apparatus}

The apparatus used was the same as that described in Experiment 1, but the signal generator and attenuator were not used. A National RS-773 tape recorder was used to transmit auditory stimuli through headphones.

\section{Subjects}

The Ss were an additional 10 male and 10 female undergraduate students in the University of Newcastle, New South Wales.

\section{Procedure}

Ss were again tested singly and the procedure described in Experiment 1 was used. When the task had been learned to criterion the two pairs of test trials were given.

Instead of a loud continuous noise, Ss were required to listen to a tape recording of $\mathrm{E}$ reciting random numbers (numbers 1-9) at 2-sec intervals while receiving the test trials. The auditory signals were presented at $95 \mathrm{~dB}$ (re $.0002 \mathrm{dyne} / \mathrm{cm}^{2}$ ). In the test trials half of the Ss received two noise trials first, followed by two no-noise trials, while the other half of the Ss followed the opposite procedure. This was done to allow for learning over test trials.

\section{Resulis}

The results for Experiment 2 are presented in Table 3. The data were analyzed by $t$ tests for the male, female, and combined samples for both the noise first and noise last conditions. It was found that the noise (numbers on tape) had no significant effect on any of the samples whether it came before or after the no-noise trials. Since in Experiment 1 noise had a significant adverse effect on the female and combined samples, it may be postulated that different types of "noise" or interference have different distraction values.

\section{DISCUSSION}

In both experiments, male and female Ss had returned to the no-noise level on the second noise trial, indicating that noise had only an initial effect. The group performance in Experiment 1 was due to the effect of the female subgroup scores.

These results could be explained by Broadbent's (1953, 1957) "distraction" view of noise, viz., that intense noise is distracting rather than disabling.

Table 2. Frequencies of correct responses for male and female $\mathrm{Ss}$ under noise and no-noise conditions.

\begin{tabular}{cccc} 
No-noise* & \multicolumn{2}{c}{ Females } \\
\hline 10 & Noise* & No-noise* & Noise* \\
14 & 10 & 13 & 6 \\
10 & 14 & 13 & 13 \\
16 & 13 & 12 & 10 \\
12 & 14 & 11 & 10 \\
12 & 14 & 11 & 5 \\
11 & 12 & 13 & 7 \\
16 & 10 & 14 & 14 \\
11 & 15 & 13 & 12 \\
15 & 12 & 12 & 13 \\
\hline
\end{tabular}

- The data represent the two Noise trials and the two No-noise trials combined respectively. 
Table 3. Data analysis for males and females under both conditions of noise in test trials.

\begin{tabular}{|c|c|c|c|c|c|c|}
\hline & \multicolumn{2}{|c|}{ Males } & \multicolumn{2}{|c|}{ Females } & \multicolumn{2}{|c|}{ Combined } \\
\hline & $\begin{array}{c}\text { Noise } \\
\text { First }\end{array}$ & $\begin{array}{c}\text { Noise } \\
\text { Lost }\end{array}$ & $\begin{array}{c}\text { Noise } \\
\text { First }\end{array}$ & $\begin{array}{r}\text { Noise } \\
\text { Last }\end{array}$ & $\begin{array}{c}\text { Noise } \\
\text { First }\end{array}$ & $\begin{array}{l}\text { Noise } \\
\text { Lost }\end{array}$ \\
\hline$\dagger$ & 1.33 & 0.18 & 0.79 & 3.00 & 1.59 & 1.34 \\
\hline$d f$ & 3 & 3 & 3 & 3 & 8 & 8 \\
\hline$P$ & $>.05$ & $>.05$ & $>.05$ & $>.05$ & $>.05$ & $>.05$ \\
\hline
\end{tabular}

Broadbent has asserted that "... the perceptual system has a limited capacity, that there is therefore competition between information from different sensory channels to enter the system, and that the degree of success of a particular stimulus in this competition depends partly on its physical intensity and partly on the length of time which has elapsed since similar information succeeded in the competition." However, we are led to hypothesize that this distracting effect of noise operates to a greater degree for females than for males and that it is only an initial effect-at least at the noise intensities used in this study.

In Experiment 1, no allowance was made for learning over test trials and any such learning should have favored an increased score under the noise condition. The fact that a significant difference was found for females between the noise and no-noise situations appears to provide even stronger evidence that females are "upset" by intense stimuli under these conditions. As males were not affected by noise in either experiment, it is unlikely that a significant difference in Experiment 1 was being masked by this procedure.

\section{References}

Broadbent, D. E. Noise, paced performance, and vigilance tasks. Brit. J. Psychol., 1953, 44, 295-303.

Broadbent, D. E. Effects of noises of high and low frequency on behaviour. Ergonomics, 1957, 1, 21-29.

Brown, R. L., Galloway, W. D., \& Gildersleeve, $\boldsymbol{K}_{\text {. }}$ R. Effects of intense noise on processing information of varying complexity. Percept. mot. Skills, 1965, 20, 749-754.

Geldard, F. A. Some neglected possibilities of communication. Science, 1960, 131, 3413, 1583-1588.

Geldard, F. A. Cutaneous channels of communication. In W. A. Rosenblith (Ed.), Sensory communication. New York: John Wiley \& Sons, Inc., 1961. Ch. 4.

Geldard, F. A. Virginia Cutaneous Project, 1948-1962. Final Report to the Office of Naval Research on Project NR-140-598. Psychological Laboratory, University of Virginia.

Gilmer, B. von Haller. Problems in cutaneous communication from psychophysics to information processing. New York. The American Foundation for the Blind, 1966.

Hodge, R. W., \& Diespecker, D. D. A two-channel signalling unit for vibrotactile experiments. Unpublished report. University of Newcastle.

Sherrick, C. E. Simple electromechanical vibration transducer. Rev. Sci. Instrum., 1965, 36, 1893-1894.

(Accepted for publication August 3, 1967.) 